\title{
Toward Centralized/Decentralized Controlled Power Flow Applying Whale Versus Genetic Optimization Algorithms
}

\author{
Nada Mamdouh Hassan, R.A.Swief, M.Z. Kamh, Hany M. Hasanien, Almoataz Youssef \\ Abdelaziz
}

\begin{abstract}
Many techniques are created in order to solve the optimal power flow issues as it is the most effective tool to get minimum cost or minimum losses under certain constraints. In this work, a smart idea is done in order to get the most optimum cost. This is done based on centralized and decentralized techniques. In general power system, decentralized technique is commonly used. However centralized technique is appeared, as this technique has a better security assessment for the system than the other. Decentralized technique based on separate the whole grid to small areas and the generating units and/or voltages on the boundaries between this area and the connected one only considered. The system is solved by two techniques; genetic algorithm (GA) and an Artificial intelligence technique called whale optimization algorithm (WOA); they are presented and utilized as optimizers in order to reach our goal. These techniques are done on IEEE 48-bus system which is consisting of two regions. These techniques are developed and simulated by using Matlab code in order to solve the OPF issue. The effect of the small voltage variations is also analyzed in order to get better optimum cost. Results demonstrate the benefits of using centralized/decentralized technique and the effect of variations of voltage.
\end{abstract}

Keywords : Centralized technique, Decentralized technique, Genetic algorithm, Optimization, Whale optimization algorithm.

\section{INTRODUCTION}

In smart grids, electrical system control is split through numerous control entities. Although each private area of the network has its responsible control entity, this control entity must take in consideration the neighboring control areas of the network as there are transmission lines interconnecting between different areas. Subsequently, the whole control entities have to collaborate with each other for obtaining effective performance, high reliability and stable smart grid. One of the major issues within the electric power system planning and control network is the performance of the optimal power flow (OPF) as it claims optimization technique with very high performance. OPF is supported tool

Revised Manuscript Received on November 15, 2019

* Correspondence Author

Nada Mamdouh Hassan, Electrical Power and Machines Department, Ain Shams University, Cairo, Egypt. Mail: nadamamdouh1988@gmail.com.

R.A.Swief, Electrical Power and Machines Department, Ain Shams University, Cairo, Egypt. Mail: rania.swief@gmail.com.

M.Z. Kamh, Electrical Power and Machines Department, Ain Shams University, Cairo, Egypt. Mail: Mohamed.zakaria@eng.asu.edu.eg.

Hany M. Hasanien, Electrical Power and Machines Department, Ain Shams University, Cairo, Egypt. Mail: hanymhasanien@gmail.com.

Almoataz Youssef Abdelaziz, Faculty of Engineering and Technology, Future University in Egypt, Cairo, Egypt. Mail: almoatazabdelaziz@hotmail.com.

in order to control the electric network taking into consideration technical and economic issues. The prime purpose for the OPF is to suppose an optimum solution for the electric power system in which the wanted objective is minimized; such as generation costs and losses; while taking into consideration security equality and inequality constraints [1].

In most smart power system grids, an OPF is carried out for the control entity taking into consideration the shared data between neighboring entities. Then, iterative decentralized or centralized algorithms are formulated to locate the most reliable and optimal operation for the electric grid. The solution of the connected areas is categorized into two approaches: decentralized or centralized solution techniques. In decentralized technique, the connected areas are considered as one area in which the control structures directly communicate and interchange information with their neighbors and all the generating units and voltage buses of the system can be changed in order to achieve the most optimum cost under certain constraints. In centralized solution technique, when the two areas get connected to each other, only the generating units and voltage buses on the boundaries can be considered. This means that the system didn't have to change all the values of its generating units and voltage buses, only the generating units and voltage buses on the boundaries can be changed in order to cover this connection, so the system security increased [2], [3].

In the past decades, a large number of classical optimization algorithms such as linear programming, non-linear programming [4] and quadratic programming effectively introduced in order to solve the OPF issues [5]. However, these algorithms having a lot of difficulties during running such as: poor convergence property, trapping into local optimum, failure to achieve global optimal solutions in a short time. These drawbacks are decreased by the appearance of a wide variety of global optimization algorithms which have been developed in order to solve the complex OPF electric power systems. These algorithms are established depending on heuristic and stochastic portions like: Particle Swarm Optimization (PSO) [6], Genetic algorithm (GA) [7], Differential evolution (DE) [8], Gravitational search algorithm (GSA) [9], Cuckoo Optimization Algorithm (COA) [10], Improved artificial bee colony (IABC) [11], Biogeography based optimization method (BBO) [12], Black hole algorithm (BH) [13], Grey Wolf Optimization (GWO) [14], Ant Lion Optimization (ALO) [1], Crow Search Algorithm (CSA) [15], and 


\section{Toward Centralized/Decentralized Controlled Power Flow Applying Whale Versus Genetic Optimization Algorithms}

Dragonfly Algorithm (DA) [16]. By all of these techniques, the system performance and accuracy have been improved.

GA suggested as a good technique for these optimization problems. These algorithms have the ability to found comprehensive implementations in solving global optimization searching cases when the closed-form optimization technique cannot be applied. The GA is more predictable to converge across the global solution in view of fact that it estimates many points in the parameter space altogether. The method may be not very sensitive to the starting points. However, it is capable to determining the global optimum solution to the OPF taking into consideration the constraints in addition to the optimum value for the objective function [17].

One of the novel utilized mechanism is the whale optimization algorithm (WOA). WOA is considered as a modern swarm brilliance-based stochastic optimization technique. Whales are characterized by exploration and exploitation which are already established in WOA. Consequently, WOA makes a very serious role to reach good implementation in order to solve the complicated optimization problems [18]. WOA has been used in a lot of applications of optimization concerns such as sizing optimization of skeletal structures [19], Semi-empirical PEM fuel cells [20], unit commitment problem [21] and economic dispatch problem [22].

In this presented paper, decentralized and centralized OPF solution and the effectiveness of each technique is illustrated by using both GA and WOA. The behavior of the system is showed in decentralized/centralized by taking into considerations the variations in voltage and power in order to get the most optimum cost. Both procedures are formulated and applied on two connecting areas according to IEEE 48 -bus test power system grid and the result are discussed. This paper consists of 6. Sections. Section 2, illustrates the general formulation of DC-OPF cost objective function containing voltages, in addition to the OPF for solving the multi-region power system network. Section 3, presents both GA and WOA and its implementation. Section 4, represents the test system under study. Simulation results are explained in section 5. Finally, conclusion and the aim of the paper clarified in section 6 .

\section{GENERAL FORMULA OF DC OPTIMAL POWER FLOW}

In this section, the drive for the DC-real power flow and the DC-OPF are illustarated and analyzed. Then connected areas OPF is presented.

\section{A. Approximation of the DC-real power flow equation}

Equation (1) illustrates the general power flow equations of the electric power system

$$
P_{k}=\sum_{j=1}^{N}\left|V_{k}\right|\left|V_{j}\right|\left(G_{k j} \cos \left(\theta_{k}-\theta_{j}\right)+B_{k j} \sin \left(\theta_{k}-\theta_{j}\right)\right)
$$

Where, $P_{k}$ is the active power generation, $\left|V_{k}\right|$ and $\left|V_{j}\right|$ are the magnitude of the voltage buses at bus $k$ and $j$ respectively, $\theta_{k}$ and $\theta_{j}$ are the voltage angles at bus $k$ and bus $j$ respectively, $G_{k j}$ is the conductance of $k j$ bus, and $B_{k j}$ is the susceptance of $k j$ bus.

Since the transmission circuit's resistance value is notably less than the reactance value. Commonly, it is when the $\mathrm{x} / \mathrm{r}$ ratio is between 2 and 10 . Therefore; as shown from (2); $G$ will be very small compared to $B$, so $G$ can be cancelled from (1) and the approximated equation for the power flow will beas shown in (3).

$$
\begin{aligned}
Y & =\frac{1}{z}=G+j B=\frac{r}{r^{2}+x^{2}}+j \frac{-x}{r^{2}+x^{2}} \\
P_{k} & =\sum_{j=1}^{N}\left|V_{k}\right|\left|V_{j}\right|\left(B_{k j} \sin \left(\theta_{k}-\theta_{j}\right)\right)
\end{aligned}
$$

Usually; under normal conditions; the differences in the angles of the voltage at two buses $k$ and $j$ connected by a circuit, is less than 10-15 degrees. Therefore, the sine function is of a small angle which can be considered the angle itself. From equation (3), when the term $j=k$ is extracted the new equation will be:

$$
\begin{gathered}
P_{k}= \\
\left|V_{k}\right|^{2} B_{k k} \sin \left(\theta_{k}-\theta_{k}\right)+\sum_{\substack{j=1 \\
j \neq k}}^{N}\left|V_{k}\right|\left|V_{j}\right|\left(B_{k j} \sin \left(\theta_{k}-\theta_{j}\right)\right)
\end{gathered}
$$

As seen the first term equal to zero. Thus, from the above discussion the final approximated power equation will be as illustrated in (5). The magnitude of voltage buses varies between 0.95 and 1.05 [23].

$$
P_{k}=\sum_{\substack{j=1 \\ j \neq i}}^{N}\left|V_{k}\right|\left|V_{j}\right|\left(B_{k j}\left(\theta_{k}-\theta_{j}\right)\right)
$$

\section{B. DC-OPF}

The main aim for the optimal power flow is to achieve the generation dispatch in order to cover the demand power as well as satisfying the constraints. The equations governing the (DC-OPF) are as showed:

$$
\begin{aligned}
& \min _{(P)} \sum_{u \in \Omega_{G}} f\left(P_{u}\right) \\
& \text { S.t. } \\
& h(x):\left\{\begin{array}{c}
P_{i}-d_{i}=\sum_{j \epsilon \Omega_{i}} \frac{\theta_{i}-\theta_{j}}{X_{i j}} \forall i \in\left\{1,2 \ldots N_{b}\right\} \\
\theta_{\text {ref }}=0
\end{array}\right. \\
& g(x):\left\{\begin{array}{c}
\underline{P_{u}} \leq P_{u} \leq \overline{P_{u}} \quad \forall u \in \Omega_{G} \\
\frac{P L_{i j}}{l_{i j}} \leq \frac{\theta_{i}-\theta_{j}}{X_{i j}} \leq \overline{P L_{i j}} \quad \forall i j \in \Omega_{L} \\
x=\{P\}
\end{array}\right.
\end{aligned}
$$

Where, $\theta$ is the angle of the voltage buses, $d_{i}$ is the demand at $i$ bus, $\Omega_{G}$ are all the generating units in the network, $\Omega_{i}$ are all the buses connected to $i$ bus, $\Omega_{L}$ are all the branches. $f(P u)$ is the cost objective function needed, the output of the generating units is denoted by $(p)$. The other two functions $h(x)$ and $g(x)$ are both the equality and inequality constraints, respectively. Balances at nodal power flow and the adjustment for the voltage angle of the reference bus to zero are guaranteed by (7). Inequality (8) secures that the output power of generating unit $u$ in addition to the power flow in transmission line $i j$ are always within their ranges [3].

\section{Electric Power System OPF for Multi- Region}

In decentralized electrical power system networks, the whole network is taken as one area and any algorithm is executed on the whole unit. However, in centralized the electrical power system grids the whole network is divided into regions and these regions are interconnected with each other through transmission lines. 
This system is called a multi-region power system network. There are two common ways to perform OPF for the multi-region networks First technique is to consider the multi-region as one network then runs the optimization technique under certain constraints and change all the generating units of the network to achieve the minimum cost. Second technique is to change only the coupling generators on the boundaries between the regions then runs the optimization technique under the same constraints to find the optimum cost. Also, smooth variations in the whole voltage or on the boundaries of the connected area in decentralized and centralized respectively, can be taken into considerations. Fig.1 illustrates the topology discussed.

\section{PRESENTATION OF OPTIMIZATION TECHNIQUES}

In this section, the used techniques for solving the OPF problem in this paper will be presented to know how they are used in order to solve this issue.

\section{A. Genetic Optimiation Algorithm}

According to the evolutionary theory of Darwin, GA is proposed and presented. it is initially utilized by Holland in 1975 , in order to get solution for the optimization problem. Originally, a set of initial random solutions is introduced which subtended to whole conditions. After that some control guidelines are encoded to make the GA able to solve OPF issue. Appearance of fitness function is introduced in order to make more resistant generations using operators of crossovers and mutations in each iteration step. The iteration process will be still going until the optimal results are obtained for OPF issue in Fig.2 [24].

\section{B. Whale Optimiation Algorithm}

WOA is introduced and developed by Mirjalili and Lewis which is dependent on the simulation of humpback whales hunting behavior [25]. There are two general mechanisms which are done during attacking: randomly tracking the victim or search for best agent, simulate the bubble net hunting strategy. Humpback whales like to hunt a set of small fish which are laying near to the surface.

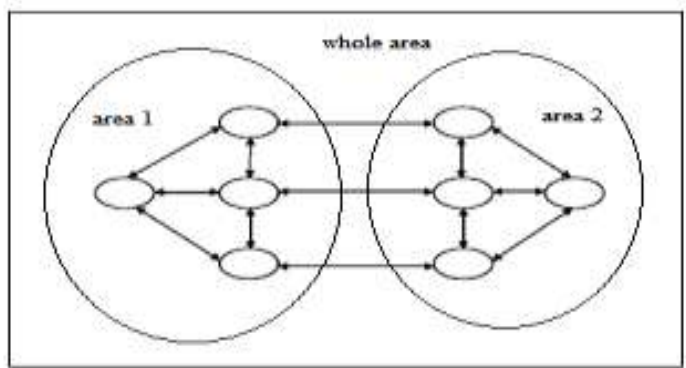

Fig. 1.Decentralized and centralized electric power system networks.

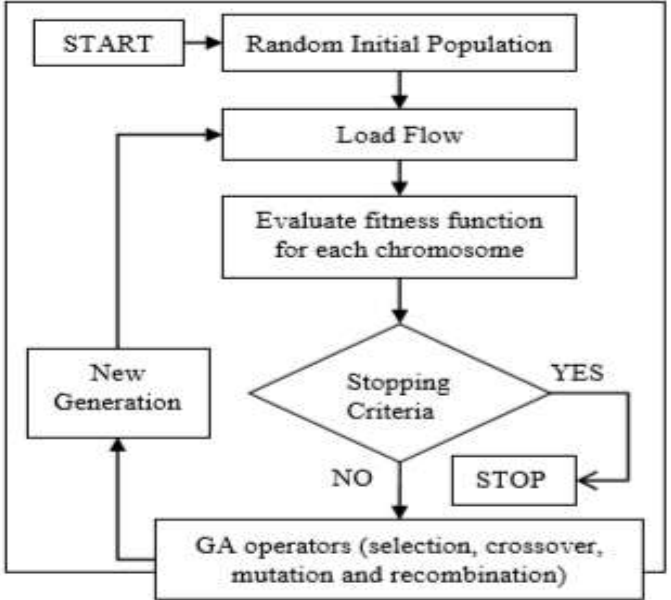

Fig. 2.General solution of OPF using GA.

They hunting the target by swimming around it inside and alongside a thin circle. Consequently, they make a winding shape and produce special blebs along the circle as shown in Fig. 3. This hunting technique is very noticeable and it is named the bubble net hunting method [21], [26].

The simulation of the WOA is presented in Fig. 4 which is mainly depend on two main stages: Exploitation stage, in which encircling victim and the bubble net hunting method are introduced. Exploration stage, in which random search for the victim is carried out. Therefore, WOA is, generally, consists of three phases.

- Encircling victim: Humpback whales have the ability to successfully find out the position of the victim and encircle them. The optimal design of the search space was unknown before, therefore WOA consider the current best candidate solution as the best one or approximately the optimum. The other search agents will therefore try to enhance their placement in direction of the best one. This manner can be introduced by:

$$
\begin{aligned}
& \vec{X}(t+1)=\overrightarrow{X^{*}}(t)-\vec{A} \cdot \vec{D} \\
& \vec{D}=\left|\vec{C} \cdot \overrightarrow{X^{*}}(t)-\vec{X}(t)\right| \\
& \vec{A}=2 \cdot \vec{a} \cdot \vec{r}-\vec{a} \\
& \vec{C}=2 \cdot \vec{r}
\end{aligned}
$$

Where, $\overrightarrow{X^{*}}$ represents the optimum result as yet, $\vec{X}$ represents the position vector, and t represents the current iteration. $\vec{A}$ and $\vec{C}$ represent the coefficient vectors which are determined from (11) and (12) respectively. The magnitude of vector $\vec{a}$ is initially 2 then diminished to $0 . \vec{r}$ represents a random vector of value between 1 and 0 [24], [27]. 


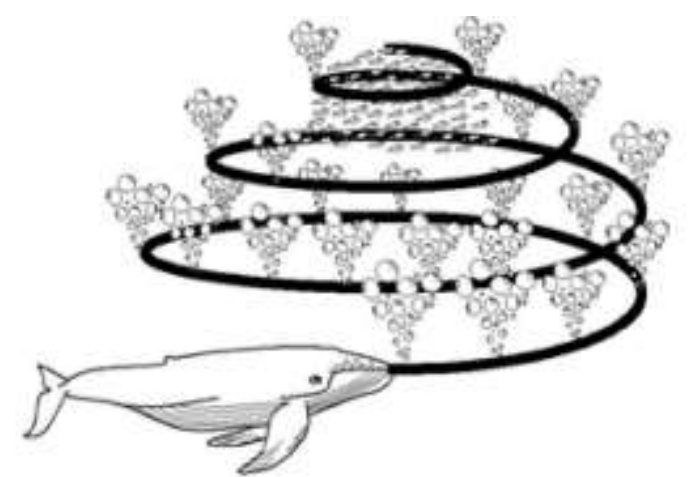

Fig. 3.The bubble net feeding of humpback whale.

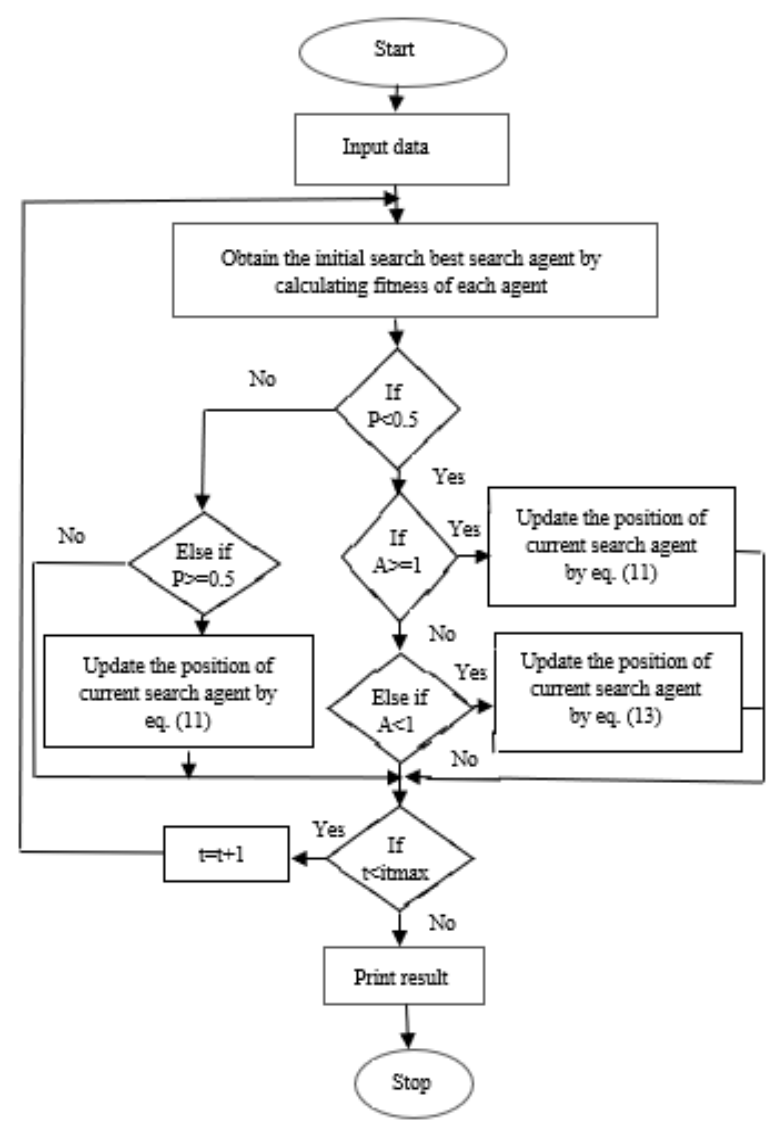

Fig. 4.A General flow chart of WOA.

- Bubble net hunting method: There are two mechanism for the hunting method

- Shrinking encircling method: to carry out this case, the value of $\vec{a}$ is decreased. Therefore, the fluctuation range of vector $\vec{A}$ will also be decreased. In this case, the value of $\vec{a}$ is decreased from 2 to 0 during the iterations. Vector $\vec{A}$ may be considered as a random value, having value within the interval $[-1,1]$. The new position of search agent will be obtained between the original position and the position of current best one.

- Spiral updating position: the spiral equation to emulate the spiral updated position is given by:

$\vec{X}(t+1)=\overrightarrow{D^{\prime}} e^{M} \cos (2 \pi t)+\overrightarrow{X^{*}}(t)$
Where,

$$
\vec{D}=\left|\overrightarrow{X^{*}}(t)-\vec{X}(t)\right|
$$

Where, $\vec{D}$ represents the distance of between the $i$-th whale and the victim (best solution), $b$ is a constant, and $t$ is a random value $[-1,1]$.

During hunting, whales swim around the victim in above two methods altogether. To choose and update whales positions $50 \%$ probability is taken for above two methods:

$$
\vec{X}(t+1)=\left\{\begin{array}{c}
\overrightarrow{X^{*}}(t)-\vec{A} \cdot \vec{D} \quad p<0.5 \\
\vec{D} e^{M} \cos (2 \pi t)+\overrightarrow{X^{*}}(t) \quad p>0.5
\end{array}\right.
$$

Where, $p$ is random value from 0 to 1 [24], [27].

- Searcg for victim: It is the exploration stage which is depending upon variance of $\vec{A}$. Humpback whales are seeking for victim randomly depending on their position. Therefore, to force the search agents to shift far away from the local whale. In this algorithm, $\vec{A}$ is used by random values greater than 1or less than -1 in order to oblige the search agent to move far away from the reference whale. The search agent position will be considered and updated to the random chosen of search agent as an alternative of the best search agent get so far. This execution helps WOA to implement global search and overcome the local optimal problem. This mechanism is expressed mathematically as follows:

$\vec{D}=\left|\vec{C} \overrightarrow{X_{\text {rand }}}-\vec{X}\right|$

$\vec{X}(t+1)=\overrightarrow{X_{\text {rand }}}-\vec{A} \cdot \vec{D}$

Where, $\overrightarrow{X_{\text {rand }}}$ represents the random position whale [24], [27].

\section{SIMULATION OF THE TEST SYSTEM UNDER STUDY}

The system used to apply the algorithms on it is the IEEE 48-bus electric system [28]. This system is composed of two regions that are connected to each other through three transmission lines as shown in Fig. 5. The elements of the first region are specified by prefix 1 , while the other elements of region 2 by prefix 2. For example, G107 indicates that it is the generating unit at bus 7 of region 1, and bus 207 indicates that it is bus number 7 in region 2 . The three transmission lines that connecting the two regions with each other are 107, 113, and 123 from region one to 203, 215, and 217 in region two respectively. Required parameters to make OPF is on IEEE 48-bus system.

\section{SIMULATION RESULT AND DISCUSSION}

In this section, the results are divided into two sections. Section 1 represents the optimization results of the decentralized technique. Section 2 represents the optimization results for the centralized technique. In both sections the study is done based on three effects which are: changing in the generating units only, variations in the voltage only, and variations in both the generating units and voltages. 
Then a detailed table for the whole system is illustrated for the two techniques. In each section results are showed according to two optimization algorithms which are: WOA and GA.

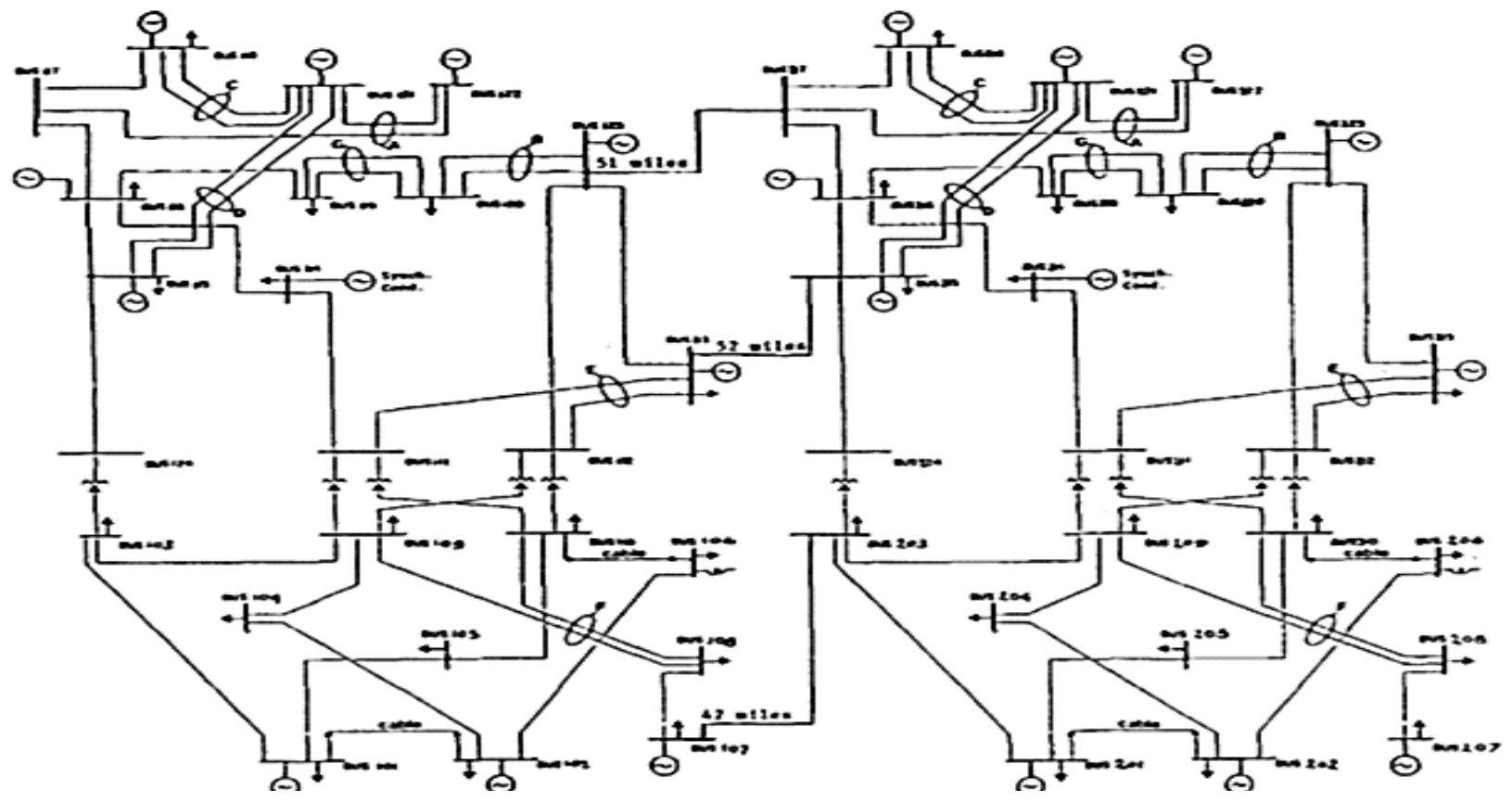

Fig. 5.IEEE 48-bus electric power system network.

\section{A. Results Based on Decentralized Technique}

In this section the system is solved based on decentralized technique. The 48-IEEE system is considered as one area by taking into account all the generating units in addition to voltage buses of the whole network. The system is studied under three effects. Firstly, the changing in the generating units are only considered, while the voltage buses are kept unity. Then, the variations of voltage buses are only considered as the voltage buses can vary from 0.95 to 1.05 , while the generating units are kept constant. Finally, the system is studied by changing both the generating units as well as the voltage buses. Results for the optimal cost is illustrated by two algorithms: WOA and GA. Table 1 illustrates the optimal cost for the three cases as well as the two algorithms used.

Table- I: Results based on decentralized technique

\begin{tabular}{|c|c|c|}
\hline \multirow{2}{*}{ Cases Studied } & \multicolumn{2}{|c|}{ Cost } \\
\hline & $G A$ & WOA \\
\hline $\begin{array}{l}\text { Variations in the generating } \\
\text { units only }\end{array}$ & 86293 & 86282 \\
\hline $\begin{array}{l}\text { Variations in the voltage } \\
\text { buses only }\end{array}$ & 160286 & 160286 \\
\hline $\begin{array}{lcr}\text { Variations } & \text { of } & \text { both } \\
\text { generating } & \text { units } & \text { and } \\
\text { voltage buses } & & \\
\end{array}$ & 86352 & 86280 \\
\hline
\end{tabular}

As shown from table I, the most optimal cost is due to the varitions in both the generating units and voltage buses. When the system is studied due to the voltage buses variatons, the cost is slightly decreased compared to the DC-power flow. Thus, the system optimal cost couldn't be found due to the variations in voltage buses only. It is also observed from the table that the cost considering WOA gives lower optimal cost than using GA. In addition, GA gives higher value by considering both generating units and voltage variations due to complicity. Thus the use of WOA based on

voltage variations and generating units gives the best result. Table II shows the detailed power flow for the whole system by considering the best result obtained.

Table- II: OPF of decentralized 48- bus system

\begin{tabular}{|c|c|c|c|c|}
\hline $\begin{array}{c}\text { Bus } \\
\text { no. }\end{array}$ & $\begin{array}{c}\mathbf{P}_{\max } \\
(\mathbf{P . U})\end{array}$ & $\begin{array}{c}\mathbf{P}_{\min } \\
(\mathbf{P . U})\end{array}$ & $\begin{array}{c}\text { Gen. } \\
\text { power } \\
\text { (P.U) }\end{array}$ & $\begin{array}{c}\text { Theta } \\
\text { (radian) }\end{array}$ \\
\hline 101 & 1.92 & 0.624 & 0.624 & -0.3329 \\
\hline 102 & 1.92 & 0.624 & 0.624 & -0.3349 \\
\hline 103 & 0 & 0 & 0 & -0.2295 \\
\hline 104 & 0 & 0 & 0 & -0.3116 \\
\hline 105 & 0 & 0 & 0 & -0.3267 \\
\hline 106 & 0 & 0 & 0 & -0.3374 \\
\hline 107 & 3 & 0.75 & 2.253 & -0.2511 \\
\hline 108 & 0 & 0 & 0 & -0.3025 \\
\hline 109 & 0 & 0 & 0 & -0.2223 \\
\hline 110 & 0 & 0 & 0 & -0.2629 \\
\hline 111 & 0 & 0 & 0 & -0.1060 \\
\hline 112 & 0 & 0 & 0 & -0.0862 \\
\hline 113 & 5.91 & 2.07 & 5.3720 & 0 \\
\hline 114 & 0 & 0 & 0 & -0.0588 \\
\hline 115 & 2.15 & 0.663 & 0.663 & 0.0523 \\
\hline 116 & 1.55 & 0.543 & 1.55 & 0.0541 \\
\hline 117 & 0 & 0 & 0 & 0.1245 \\
\hline 118 & 4 & 1 & 4 & 0.1455 \\
\hline 119 & 0 & 0 & 0 & 0.0495 \\
\hline 120 & 0 & 0 & 0 & 0.0784 \\
\hline 121 & 4 & 1 & 4 & 0.1566 \\
\hline 122 & 3 & 0.6 & 3 & 0.2567 \\
\hline & & & & \\
\hline
\end{tabular}




\section{Algorithms}

\begin{tabular}{|c|c|c|c|c|}
\hline $\begin{array}{l}\text { Bus } \\
\text { no. }\end{array}$ & $\begin{array}{l}\mathbf{P}_{\max } \\
\text { (P.U) }\end{array}$ & $\begin{array}{c}\mathbf{P}_{\min } \\
\text { (P.U) }\end{array}$ & $\begin{array}{c}\text { Gen. } \\
\text { power } \\
\text { (P.U) }\end{array}$ & $\begin{array}{l}\text { Theta } \\
\text { (radian) }\end{array}$ \\
\hline 123 & 6.6 & 2.486 & 6.6 & 0.1067 \\
\hline 124 & 0 & 0 & 0 & -0.0554 \\
\hline 201 & 1.92 & 0.624 & 0.624 & -0.3685 \\
\hline 202 & 1.92 & 0.624 & 0.624 & -0.3706 \\
\hline 203 & 0 & 0 & 0 & -0.2625 \\
\hline 204 & 0 & 0 & 0 & -0.3475 \\
\hline 205 & 0 & 0 & 0 & -0.3629 \\
\hline 206 & 0 & 0 & 0 & -0.3739 \\
\hline 207 & 3 & 0.75 & 2.286 & -0.2730 \\
\hline 208 & 0 & 0 & 0 & -0.3307 \\
\hline 209 & 0 & 0 & 0 & -0.2584 \\
\hline 210 & 0 & 0 & 0 & -0.2997 \\
\hline 211 & 0 & 0 & 0 & -0.1451 \\
\hline 212 & 0 & 0 & 0 & -0.1258 \\
\hline 213 & 5.91 & 2.07 & 5.169 & -0.0485 \\
\hline 214 & 0 & 0 & 0 & -0.0963 \\
\hline 215 & 2.15 & 0.663 & 0.663 & 0.0149 \\
\hline 216 & 1.55 & 0.543 & 1.55 & 0.0180 \\
\hline 217 & 0 & 0 & 0 & 0.0914 \\
\hline 218 & 4 & 1 & 4 & 0.1113 \\
\hline 219 & 0 & 0 & 0 & 0.0131 \\
\hline 220 & 0 & 0 & 0 & 0.0417 \\
\hline 221 & 4 & 1 & 4 & 0.1215 \\
\hline 222 & 3 & 0.6 & 3 & 0.2224 \\
\hline 223 & 6.6 & 2.486 & 6.6 & 0.0700 \\
\hline 224 & 0 & 0 & 0 & -0.0911 \\
\hline
\end{tabular}

using WOA. When the system is studied due to the voltage buses variatons, the cost is slightly decreased from the DCpower flow case. Thus, the system optimal cost couldn't be found due to the variations in voltage buses only. It is also observed from the table that the cost considering WOA gives lower optimal cost than using GA. Thus the use of WOA based on voltage variations and generating units gives the best result. Table IV shows the detailed power flow for the whole system by considering the best result obtained.

Table- IV: OPF of decentralized 48- bus system

\begin{tabular}{|c|c|c|c|c|}
\hline $\begin{array}{l}\text { Bus } \\
\text { no. }\end{array}$ & $\begin{array}{c}\mathbf{P}_{\max } \\
\text { (P.U) }\end{array}$ & $\begin{array}{c}\mathbf{P}_{\min } \\
(\mathbf{P . U})\end{array}$ & $\begin{array}{c}\text { Gen. } \\
\text { power } \\
\text { (P.U) }\end{array}$ & $\begin{array}{l}\text { Theta } \\
\text { (radian) }\end{array}$ \\
\hline 101 & 1.92 & 0.624 & 1.72 & -0.1540 \\
\hline 102 & 1.92 & 0.624 & 1.72 & -0.1558 \\
\hline 103 & 0 & 0 & 0 & -0.1269 \\
\hline 104 & 0 & 0 & 0 & -0.2032 \\
\hline 105 & 0 & 0 & 0 & -0.2078 \\
\hline 106 & 0 & 0 & 0 & -0.2530 \\
\hline 107 & 3 & 0.75 & 1.1694 & -0.3019 \\
\hline 108 & 0 & 0 & 0 & -0.3118 \\
\hline 109 & 0 & 0 & 0 & -0.1652 \\
\hline 110 & 0 & 0 & 0 & -0.2014 \\
\hline 111 & 0 & 0 & 0 & -0.0582 \\
\hline 112 & 0 & 0 & 0 & -0.0472 \\
\hline 113 & 5.91 & 2.07 & 2.5846 & 0 \\
\hline 114 & 0 & 0 & 0 & 0.0153 \\
\hline 115 & 2.15 & 0.663 & 2.15 & 0.1786 \\
\hline 116 & 1.55 & 0.543 & 1.55 & 0.1592 \\
\hline 117 & 0 & 0 & 0 & 0.2440 \\
\hline 118 & 4 & 1 & 4 & 0.2708 \\
\hline 119 & 0 & 0 & 0 & 0.1302 \\
\hline 120 & 0 & 0 & 0 & 0.1413 \\
\hline 121 & 4 & 1 & 4 & 0.2862 \\
\hline 122 & 3 & 0.6 & 3 & 0.3934 \\
\hline 123 & 6.6 & 2.486 & 6.6 & 0.1602 \\
\hline 124 & 0 & 0 & 0 & 0.0618 \\
\hline 201 & 1.92 & 0.624 & 1.72 & -0.2546 \\
\hline 202 & 1.92 & 0.624 & 1.72 & -0.2554 \\
\hline 203 & 0 & 0 & 0 & -0.2601 \\
\hline 204 & 0 & 0 & 0 & -0.2998 \\
\hline 205 & 0 & 0 & 0 & -0.3017 \\
\hline 206 & 0 & 0 & 0 & -0.3430 \\
\hline 207 & 3 & 0.75 & 2.4 & -0.2495 \\
\hline 208 & 0 & 0 & 0 & -0.3201 \\
\hline 209 & 0 & 0 & 0 & -0.2595 \\
\hline 210 & 0 & 0 & 0 & -0.2883 \\
\hline 211 & 0 & 0 & 0 & -0.1626 \\
\hline 212 & 0 & 0 & 0 & -0.1456 \\
\hline 213 & 5.91 & 2.07 & 2.853 & -0.1033 \\
\hline 214 & 0 & 0 & 0 & -0.1039 \\
\hline 215 & 2.15 & 0.663 & 0.663 & 0.0264 \\
\hline 216 & 1.55 & 0.543 & 1.5 & \\
\hline
\end{tabular}




\begin{tabular}{|c|c|c|c|c|}
\hline $\begin{array}{c}\text { Bus } \\
\text { no. }\end{array}$ & $\begin{array}{c}\mathbf{P}_{\max } \\
(\mathbf{P . U})\end{array}$ & $\begin{array}{c}\mathbf{P}_{\min } \\
(\mathbf{P . U})\end{array}$ & $\begin{array}{c}\text { Gen. } \\
\text { power } \\
\text { (P.U) }\end{array}$ & $\begin{array}{c}\text { Theta } \\
\text { (radian) }\end{array}$ \\
\hline 217 & 0 & 0 & 0 & 0.1184 \\
\hline 218 & 4 & 1 & 4 & 0.1410 \\
\hline 219 & 0 & 0 & 0 & 0.0127 \\
\hline 220 & 0 & 0 & 0 & 0.0368 \\
\hline 221 & 4 & 1 & 4 & 0.1516 \\
\hline 222 & 3 & 0.6 & 3 & 0.2652 \\
\hline 223 & 6.6 & 2.486 & 6.6 & 0.0638 \\
\hline 224 & 0 & 0 & 0 & -0.0831 \\
\hline
\end{tabular}

\section{Comparison between Different Systems}

Table-V: Cost under different cases

\begin{tabular}{|c|c|}
\hline Cases Studied & Cost by WOA \\
\hline DC- power flow & 160286 \\
\hline Decentralized OPF & 86280 \\
\hline Centralized OPF & 144392 \\
\hline
\end{tabular}

As shwon from table $\mathrm{V}$, the cost due to optimizatation is lower than the DC-power flow. In addition, the cost considering one region is lower than considering two region, however the differnce between them is not too large. So, the system can be solved by considering only the boundaries in order not to change the whole system to increase the stability of the system and lowering the changes.

\section{CONCLUSION}

This paper analyzes optimization techniques based on the DC-OPF for solving decentralized and centralized areas. The network introduced is solved by using GA and WOA techniques to reach the minimum production cost. Procedures are formulated and applied on two connecting areas according to IEEE 48-bus test power system grid. The system is solved in decentralized mode and also solved by only considering the boundaries between the two regions.

Case studies are accomplished via GA and WOA: the centralized and decentralized system is studied in order to obtain the most optimal cost by taking in considerations the generating unit's changes. The results showed that WOA gives lower cost than GA as it is improved and newest technique. In addition, the system is studied based on the voltage variations. Since the changes in volt is slightly small the optimal cost did not change too much. Finally, the system is studied under the effect of both voltage variations as well as changing of generating units. Due to these both effects the system gives the most optimal cost among the other cases. The results are introduced and demonstrate an encouraging performance for using the last case. In addition, results showed that, the changes in the generating units on the boundaries only of two connecting areas, give lower cost compared to DC-power flow, so it can be used as it introduces higher security technique.

\section{REFERENCES}

1. H. Ouafa, S. Linda, and B. Tarek, "Optimal power flow with four conflicting objective functions using multiobjective ant lion algorithm: a case study of the algerian electrical network," Iranian journal of electrical and electronic engineering 01, vol. 15, no. 1,2019, pp. 94-113.

2. Hany M. Hasanien, "Whale optimisation algorithm for automatic generation control of interconnected modern power systems including renewable energy sources", IET Generation, Transmission \& Distribution, vol. 12, no. 3, Feb. 2018, pp. 607-614.

3. K. Amin, M. Javad, G. Junyao, C. Sambuddha, B. Masoud, H. Gabriela et al., "Toward distributed/decentralized dc optimal power flow implementation in future electric power systems,' IEEE Transactions on smart grid, vol. 9, no. 4,2018, pp. 2574-2594.

4. H. Habibollahzadeh, G.X. Luo, and A. Semlyen, "Hydrothermal optimal power flow based on a combined linear and nonlinear programming methodology,' IEEE transaction power system, vol. 4, no. 2,1989, pp. 530-537.

5. S. Zhang and M.R. Irving, “ Enhanced Newton-Raphson algorithm for normal, controlled and optimal power flow solutions using column exchange techniques,' IEE generation transmission distribution, vol. 141, no. 6,1994, pp. 647-657.

6. Z. Yujiao and S. Yanguang, "'Solving multiobjective optimal reactive power dispatch using improved multiobjective particle swarm optimization,"'The 26th Chinese control and decision conference, Changsha, China,2014, pp. 1010-1015.

7. A. Saleh,T.A. Fares, and M. Joydeep, "Opposition-based elitist real genetic algorithm for optimal power flow," North american power symposium (NAPS), Denver, CO, USA,2016, pp. 1-6.

8. P.B. Partha, P.N. Suganthan, R. Mallipeddi, and A.J.A. Gihan, “‘ Optimal power flow solutions using differential evolution algorithm integrated with effective constraint handling techniques, ' Engineering applications of artificial intelligence, Vol. 68,2018, pp. 81-100.

9. R. Jordan, J. Miroljub, A. Nebojsa, and K. Dardan, ' 'Optimal power flow for distribution networks using gravitational search algorithm," Electric engineering, vol. 96,2014, pp. 335-345.

10. D. Le Anh and D. Vo Ngoc, "Application of Cuckoo search algorithm for optimal power flow in power system,' GMSARN International journal, vol. 9,2015, pp. 45-50.

11. B. Wenlei, E. Ibrahim, and Y.L. Kwang, "An improved artificial bee colony optimization algorithm based on orthogonal learning for optimal power flow problem,'” Control engineering practice, Vol. 61,2017, pp. 163-172 (April 2017).

12. H. Ouafa, S. Linda, and B. Tarek, "Biogeography based optimization approach for solving optimal power flow problem," International journal of hybrid information technology, Vol.6, No.5,2013, pp. 183-196.

13. H. Abdolreza, "Black hole: A new heuristic optimization approach for data clustering," Information sciences, Vol. 222, 2013, pp. 175-184.

14. T. Kiran_and D.M.V. Kumar, "Grey wolf optimization algorithm based dynamic security constrained optimal power flow,' National power systems conference (NPSC), Bhubaneswar, India, 2016.

15. S. Anulekha, B. Aniruddha, Priyanath D., and K.C. Ajoy, "Crow search algorithm for solving optimal power flow problem,', Second International conference on electrical, computer and communication technologies (ICECCT), Coimbatore, India, February 2017.

16. M. Seyedali, "Dragonfly algorithm: a new meta-heuristic optimization technique for solving single -objective, discrete, and multi-objective problems," Neural computing and applications, Vol. 27, No. 4,2016, pp. 1053-1073.

17. SatyendraSingh and K.S. Verma, "Optimal power flow using genetic algorithm and particle swarm optimization," IOSR journal of engineering (IOSRJEN), vol. 2, Issue 1,2012, pp. 046-049.

18. P. Dharmbir, M. Aparajita, S. Gauri, and M. Vivekananda, "Application of chaotic whale optimization algorithm for transient stability constrained optimal power flow,' IET science, measurement and technology, Vol. 11, Issue 8, 2017, pp. 1002-1013.

19. A. Kaveh, M.I. Ghazaan, "Enhanced whale optimization algorithm for sizing optimization of skeletal structures, Mechanics based design of structures and machines,' An international journal, vol. 45, Issue 3 , 2017, pp. 345-362.

20. A. A. El-Fergany, Hany M. Hasanien and, A. M. Agwa, "Semi-empirical PEM fuel cells model using whale optimization algorithm", Energy Conversion and Management, vol. 201, no. 112197, December 2019, pp. 1-11,.

21. L. Dilip, N.T. Indrajit, J. Pradeep, and K. Arvind, "A whale optimization algorithm approach for unit commitment problem solution," National conferance on advancements in electrical and power electronics engineering (AEPEE 2016), Morbi, India, 2016.

22. J.T. Haider, "Study of the economic dispatch problem on IEEE 30 bus system using whale optimization algorithm,' International journal of 


\section{Toward Centralized/Decentralized Controlled Power Flow Applying Whale Versus Genetic Optimization}

Algorithms

engineering technology and sciences (IJETS), vol. 5, no. 1,2016, pp. 11-18.

23. G. Arman, K. Mehdi, S. Andrew, and M.H.N. Seyed, "DC Optimal Power Flow through the Linear Programming - in Context of Smart Grid," 24th Southern African Universities Power Engineering Conference, Vereeniging, South Africa January 2016.

24. I. C. Rengin, E.T. Belgin, "Heuristic methods to solve optimal power flow problem," IU-JEEE, Vol. 13, no. 2, 2013, pp. 1653-1659.

25. F. Islam, Hany M. Hasanien, A. Al-Durra, and S.M.Muyeen, "A New Control Strategy for Smoothing of Wind Farm Output using Short-Term Ahead Wind Speed Prediction and Flywheel Energy Storage System", in the proceeding of American Control Conference, ACC, 27-29June 2012, Montreal, Canada.

26. Hany M. Hasanien, "Performance improvement of photovoltaic power systems using an optimal control strategy based on whale optimization algorithm”, Electric Power Systems Research, vol. 157, April 2018, pp. 168-176.

27. M. H. Qais, Hany M. Hasanien and S. Alghuwainem, "Whale optimization algorithm-based Sugeno fuzzy logic controller for fault ride-through improvement of grid-connected variable speed wind generators", Engineering Applications of Artificial Intelligence, vol. 87, 103328, January 2020, pp. 1-13.

28. C. Grigg, P. Wong, P. Albrecht, R. Allan, M. Bhavaraju, R. Billinton, et al. " "The IEEE reliability test system-1996. A report prepared by the reliability test system task force of the application of probability methods subcommittee,"' IEEE transactions on tower systems, vol. 14, no. 3,1999 , pp. $1010-1020$.

\section{AUTHORS PROFILE}

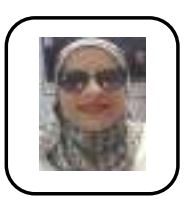

Nada Mamdouh Hassan got the B.Sc. and M.Sc degrees from Ain Shams University, Cairo, Egypt, in 2011 and 2017 respectively. Both in electrical power and machines engineering. M.Sc. was in the area of Renewable energy. She has publications in international conferences. She is currently a teaching Assistant with the department of Electrical Power and Machines, higher institute of engineering and technology- 5-th settlement, Cairo, Egypt. Her Fields of Interests lie in Power System Analysis, Control, and Renewable Energy.

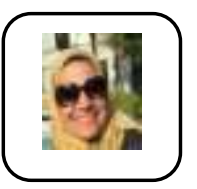

R.A.Swief Got her B.Sc., M.Sc., Ph.D, and Associate Professor from Ain Shams University, Cairo, Egypt on $1998,2004,2010$, and 2018 respectively. Ph.D was in the area of Deregulated market and price load market relations. Contributed in many paper nationally and internationally. She has many publications in local and international conferences. Supervised many Ph.D and Master degrees in the areas of Smart grid, Protection, Deregulated market and Renewable Energy. Her Fields of Interests lie in Power System Analysis, Planning, and Renewable Energy.

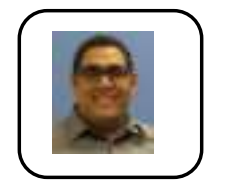

Mohamed Zakaria Kamh received the B.Sc. (Honors) and M.Sc. degrees from Ain Shams University, Cairo, Egypt, in 2003 and 2007 respectively, both in electrical power and machines engineering, and the Ph.D. degree in electrical engineering from University of Toronto, Toronto, ON, Canada, in 2011. He is currently an Assistant Professor with the department of Electrical Power and Machines, Ain Shams University, Cairo, Egypt, and serves as the Technical Adviser for the Ministry of Electricity and Renewable Energy for Transmission Network planning and operation. He is a registered Professional Engineer in Egypt, as well as with the Provinces of Alberta and Ontario, Canada where he served for more than a decade as a utility leader in the fields of transmission system planning, operation, and engineering. His research interests include power system planning and operation, power electronics, distributed and renewable energy resources, smart grids, and application of artificial intelligence in the aforementioned fields.

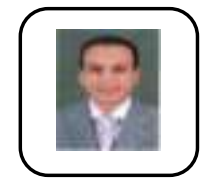

Hany M. Hasanien received his B.Sc., M.Sc. and Ph.D. degrees in Electrical Engineering from Ain Shams University, Faculty of Engineering, Cairo, Egypt, in 1999, 2004, and 2007, respectively. From 2008 to 2011, he was a Joint Researcher with Kitami Institute of Technology,

Almoataz Y. Abdelaziz received the B.Sc. and M.Sc degrees in electrical engineering from Ain Shams University, Cairo, Egypt, in 1985 and 1990, respectively, and the $\mathrm{Ph} . \mathrm{D}$. degree in electrical engineering according to the channel system between Ain Shams University, Egypt, and Brunel University, U.K., in 1996.

$\mathrm{He}$ is a professor of electrical power engineering at Ain Shams University since 2007. He has authored or coauthored more than 400 refereed journal and conference papers, 25 book chapters and 3 edited books with Elsevier and Springer in his research areas which include the applications of artificial intelligence, evolutionary and heuristic optimization techniques to power systems power system operation, planning, and control.

Dr. Abdelaziz is the chairman of IEEE Education Society chapter in Egypt, a senior editor of Ain Shams Engineering Journal, editor of Electric Power Components \& Systems Journal, Editorial Board member, Editor, Associate Editor, and Editorial Advisory Board member for many international journals.

$\mathrm{He}$ is also a senior member in IEEE, a member in IET and the Egyptian Sub-Committees of IEC and CIGRE'. He has been awarded many prizes for distinct researches and for international publishing from Ain Shams University and Future University in Egypt. 